\title{
Modeling the dynamic response of conduits
}

\author{
V I Hanby ${ }^{1}$, J A Wright ${ }^{2}$, D W Fletcher ${ }^{3}$ and D N T Jones ${ }^{3}$
}

1. Institute of Energy and Sustainable Development, De Montfort University, UK.

2. Department of Civil and Building Engineering, Loughborough University, UK.

3. Motor Industry Research Association, Nuneaton, UK.

\begin{abstract}
A method for the dynamic modeling of a fluid conduit is proposed, based on its discretization into a sequence of well-mixed flow nodes. This enables the time delay produced by the fluid flow to be simply modeled in any time domain simulation. An optimal level of discretization, based on the residence time distribution produced by fully developed turbulent flow, is presented. The model is capable of calculating the response to changes in flow rate, fluid inlet temperature and species concentration.

The thermal response is based on a second order model for each node, taking account of the thermal capacitance of the fluid and of the conduit inner wall. It is demonstrated that the model predicts a time delay, then a rapid initial response due to flow effects, followed by slower dynamics controlled by the thermal inertia of the walls. An inter-model comparison of output for a prototype duct is made with three published models and an empirical validation is reported.
\end{abstract}

\section{Introduction}

The dynamic simulation of duct and pipe behaviour has been studied less intensively than that of most HVAC components. This may be due in part to the comparatively limited number of situations in which the dynamic characteristics of flow and heat transfer are important. Some examples of situations in which dynamic effects are significant are

- local loop control, where the sensor is remote from the actuator;

- situations where the dynamics of the distribution of some hazardous material (e.g. smoke) in a ductwork system is important;

- situations where short-term thermal dynamics are significant, such as boiler flue systems and automobile exhausts.

A thorough survey of existing dynamic models has recently been published (Bourdouxhe et al., 1998) which divides models into three categories: those governed by partial differential equations, lumped parameter models (ordinary differential equations) and filters, in which dynamics are added to steady-state models via a first-order Hammerstein paradigm. The focal point of all these models was thermal response, the flow dynamics being considered only as a time delay. The review points out that the models surveyed generally neglect the fluid thermal capacitance, hence are not suitable for liquid flows, assume a fixed fluid mass flow rate and do not treat the issue of time delay in a realistic manner. 
A starting point for several model implementations was the classical transfer function approach (Tobias, 1973). Working from partial differential equations representing energy balances on the fluid and conduit material, a transfer function giving the temperature response at the outlet of the duct was obtained. The form of this function made it difficult to transpose to the time domain, so a simplified function was recommended for practical use. It should be noted that Tobias' model does not take into account the longitudinal temperature distribution of the conduit, which limits its range of application.

The implementation of duct models for the modular simulation program HVACSIM+ (Park et al., 1985) has been described by Clark et al (1985). The paper described a simplified model based on the filter approach and a lumped parameter model obtained by expressing Tobias' simplified transfer function in the time domain. Both models required a representation of a time delay caused by the transport time of fluid in the duct. This can be difficult to accommodate in many simulation solvers, whether a fixed or variable time step is used. The time delay is generally taken as

$$
t_{d}=\frac{V}{\dot{\nu}}
$$

In the method of representing time delay described by Clark, the temperature distribution is given by a fifth order polynomial in distance with time-dependent coefficients, determined by Gaussian elimination at each timestep. The duct is discretized into five sections in this model.

$$
T(x, t)=\sum_{i=0}^{i=5} a_{i}(t) x^{i}
$$

The simplified model superimposed a first order dynamic response onto this time delay

$$
\frac{d T_{\text {out }}}{d t}=\frac{T_{s s}-T_{\text {out }}}{t_{c}}
$$

where the time constant is given by

$$
t_{c}=\left(\frac{h_{i}}{h_{i}+h_{o}}\right) \frac{C_{w}}{\dot{m} c_{p}}
$$

Clark also derived a time-domain transformation of Tobias's approximate transfer function. This model has three components: a time delay as per equation 2, a step rise in temperature followed by a first order temperature rise. For a unit step input:

$$
T_{\text {out }}=\lambda+(1-\lambda)\left(1-e^{-\frac{t}{t_{c}^{T}}}\right)
$$

for $t>t_{d}, T_{\text {out }}=0$ otherwise

where 


$$
\begin{aligned}
& \lambda=e^{-\frac{h_{i} A}{\dot{m} c_{p}}} \\
& t_{c}^{\prime}=t_{c} e^{-\alpha}
\end{aligned}
$$

and

$$
\alpha=\frac{U A H_{i}}{2 \dot{m} c_{p} h_{o}}
$$

A duct model was developed (Type 31) and implemented in the simulation program TRNSYS (Klein 1996). The fluid flow component of the model consists of defining segments of the fluid of variable size, each segment of fluid having a first-order differential equation to model its temperature dynamics. The temperature history of the leaving fluid is thus defined by the segments that are pushed out of the duct by the entering fluid. This model does not take into account the thermal capacitance of the conduit.

The approach developed in this paper is to represent the fluid flow element of the simulation by using a connected sequence of well-mixed nodes. Each fluid node, which is defined by a single temperature, then exchanges heat with its local conduit walls. This method was briefly outlined by Escudie and Laret (1994) and it has a number of advantages. Firstly, by using simple firstorder dynamics, problems associated with explicit time delay modeling are avoided. Secondly, the thermal capacity of both fluid and conduit wall are easily accounted for, making the model equally applicable to pipes as well as ducts.

The method described here is based on discretization of the conduit into a series of well-mixed zones and establishes the optimum number of zones to use, based on the criterion of matching as closely as possible the residence time distribution which occurs in the prototype conduit. This makes the model equally suitable for studying both the thermal response and the fluid transport behaviour of duct or pipe systems. In this paper, the prototype residence time distribution has been obtained using a published analytical expression. However it should be noted that the approach would be equally suitable if the residence time distribution were to be obtained by other means such as CFD simulation or by experimental measurement.

\section{Residence time distribution}

\subsection{The $\mathrm{F}$ diagram}

A convenient way of representing residence time distribution (RTD) is given by considering the following: at time $t=0$ a passive fluid property in a steady-flow system undergoes a step change (for example, the colour could change from clear to red). The variable $F(t)$ represents the fraction of red material in the outgoing fluid at time $t$. The analysis is simplified if we use dimensionless time, given by 


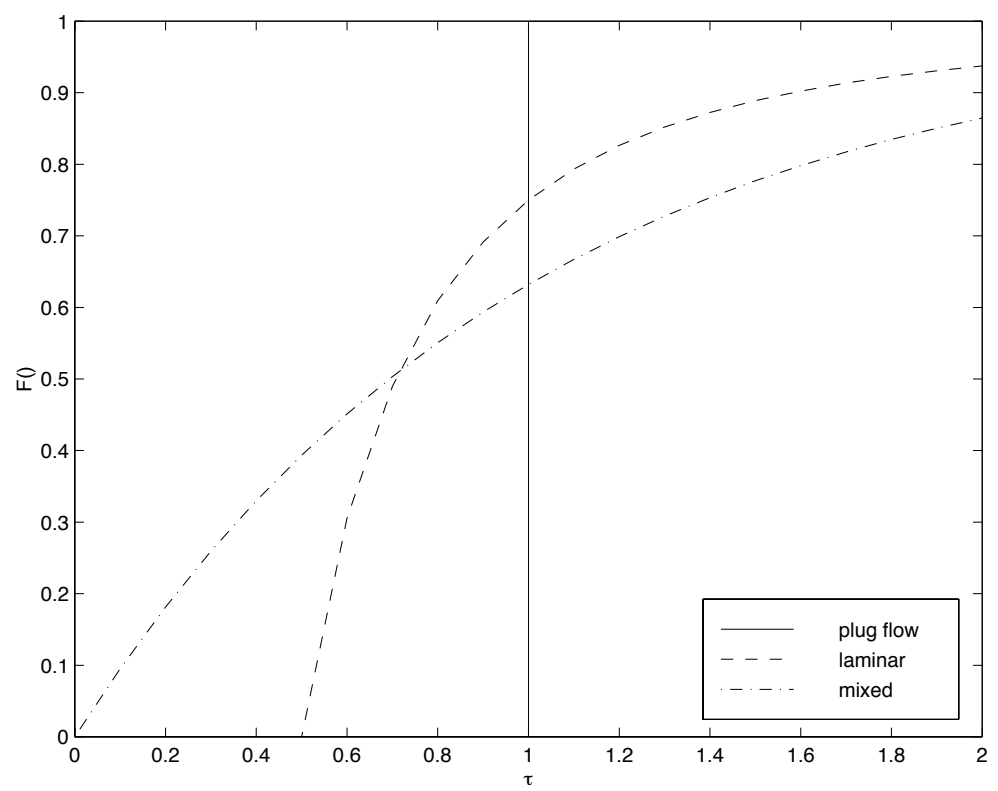

Figure 1: F diagrams for plug, laminar and well-mixed flow

$$
\tau=\frac{\dot{\nu} t}{V}
$$

The actual shape of the F-diagram in a duct or pipe depends primarily on the velocity profile. The plug flow assumption is predicated on a flat velocity profile across the duct, hence all the red fluid elements arrive at the exit at the same time. If a velocity profile is considered, then the faster moving elements near the centerline will arrive more quickly than the average. In the case of laminar flow, the maximum velocity is double that of the mean fluid velocity, hence red material would first appear in the exit flow at $\tau=0.5$. The F-diagrams for the limiting conditions of plug flow, well-mixed flow (in which an entering fluid element is instantaneously dispersed throughout the volume) and laminar flow are shown in Fig 1.

Neglecting mixing, an F function can be obtained by integration of the velocity profile. For laminar flow this easily shown to give

$$
F(\tau)=1-\frac{1}{4 \tau^{2}} \quad \tau>0.5
$$

For any given situation, the RTD can be obtained by three means:

1. direct experiment measurement using a tracer fluid;

2. numerical modeling using CFD techniques;

3. a closed-form equation, taking into account velocity profile and diffusivity (to incorporate the effects of mixing). 
There are very few reported experimental investigations relating to the RTD of turbulent flow in a conduit, and the experimental difficulties of obtaining reliable measurements are not trivial. The approach described in this paper is based on matching the actual RTD as closely as possible by using a sequence of well-mixed nodes. As an example of the implementation of this approach, the actual RTD is obtained by the last of these methods, the assumption of a fully-developed turbulent velocity profile.

\subsection{Turbulent velocity profile-based F function for a circular conduit}

A power law radial velocity profile is assumed:

$$
u_{r}=u_{\max }(1-r)^{\frac{1}{n}}
$$

The maximum velocity $u_{\max }$ is related to the mean $\bar{u}$ by

$$
\begin{gathered}
\bar{u}=2 \frac{u_{\max }}{\left(\frac{1}{n}+1\right)\left(\frac{1}{n}+2\right)} \\
n= \begin{cases}7 & 2000<N_{R e}<10^{5} \\
8 & N_{R e}>10^{5}\end{cases}
\end{gathered}
$$

A correction factor allows for the effects of eddy diffusivity (Danckwerts 1953):

$$
\beta=\frac{(n-1)^{2} R}{0.32 n L}
$$

The combined effects of velocity profile and diffusion give the following expression for $F$

$$
F(\tau)=1+\frac{n+1-\tau^{n}(2 n-1)}{n\left(\tau^{n}\right)^{2} \tau}+\frac{\beta(n+1)(2 n+1)^{2}\left(2 \tau^{n}-1\right)\left(\tau^{n}-1\right)^{2}}{4 n^{2}\left(\tau^{n}\right)^{4}}
$$

The first two terms define the RTD by integration (with respect to time and radial position) of the velocity profile while the third term (containing $\beta$ ) is the diffusive correction factor. This $\mathrm{F}$ diagram is shown in Figure 2 for $n=7$ and $\beta=0.01$. A similar expression was derived by Bosworth (1949) and compared with experimental results for water flow in a pipe which showed that equation 11 tended to over-predict the extent of longitudinal mixing. It should be noted that the length : diameter ratio of the duct only affects the diffusivity correction term. 

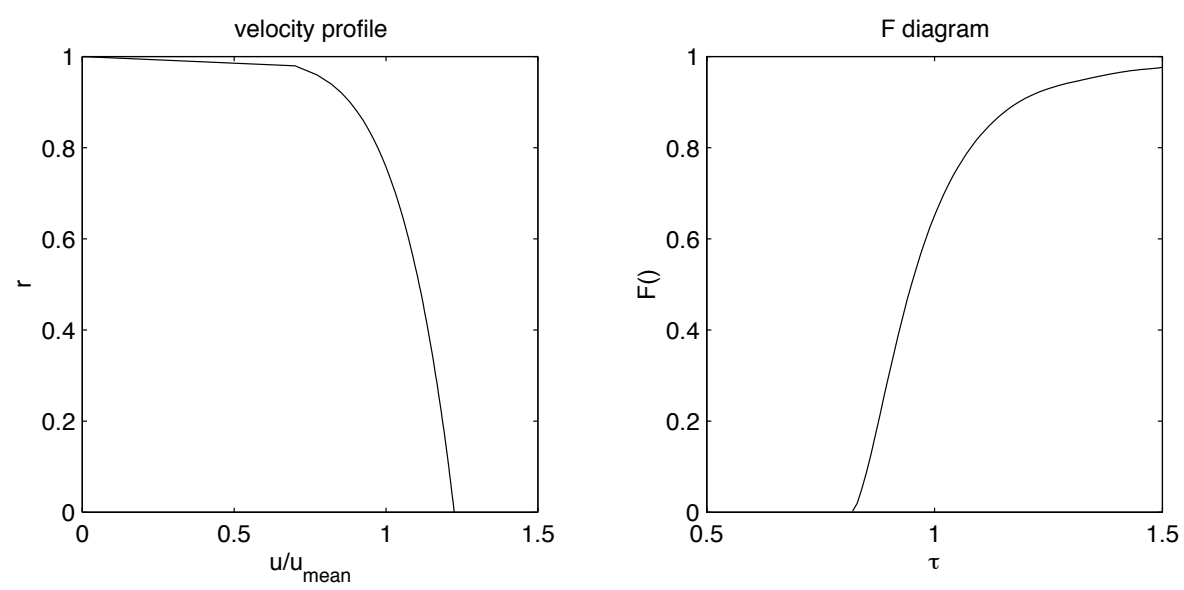

Figure 2: Velocity profile and F-diagram for turbulent flow in conduit

\section{Well-mixed nodal model}

\subsection{Response to flow disturbances}

The approach described in this paper is to obtain an approximation to the benchmark F diagram (here taken as that given by equation 11) by defining a model consisting of a number of well-mixed nodes in series.

For a step change in input the response of a single node is an exponential rise

$$
F(\tau)=1-e^{-\tau}
$$

If we consider a number of such nodes $(i)$ in series such that the volume of each node is $V / i$, then it can easily be shown that the resulting $\mathrm{F}$ diagram is given by

$$
F(\tau)=1-e^{-i \tau}\left[1+i \tau+\frac{1}{2 !}(i \tau)^{2}+\ldots \frac{1}{(i-1) !}(i \tau)^{(i-1)}\right]
$$

As the number of nodes is increased, the order of the response rises and as the number of nodes approaches infinity the response approaches that of plug flow. Figure 3 shows a plot of equation 13 for 20, and 80 nodes in series, together with the analytical result given by equation 11 .

The optimum number of nodes was established by evaluating the area enclosed between the $\mathrm{F}$ diagram for that number of nodes and the $\mathrm{F}$ diagram of the benchmark model over the range 


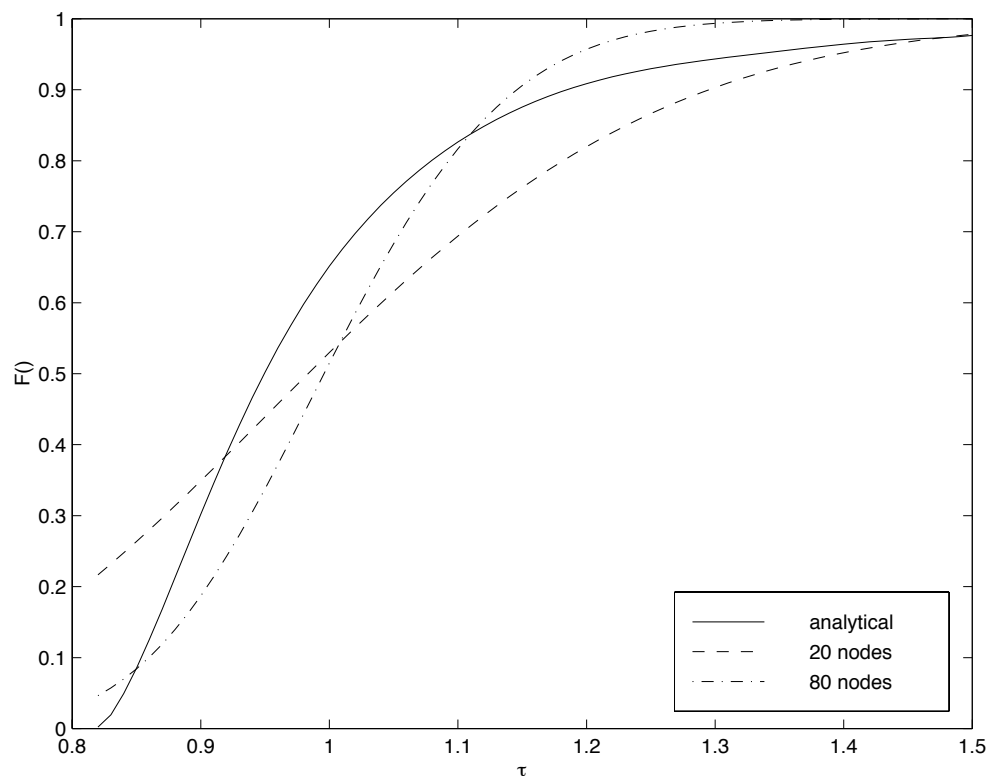

Figure 3: F-diagrams for well-mixed nodes in series

$0.8<\tau<1.5$. The results are shown in Figure 4, which indicates that the optimum number of nodes is 46 , but that if a reduction in problem size is required for computational constraints then 20 gives a reasonable approximation.

It is of interest to note that the optimal number of nodes is independent of any conduit properties with regard to the radial velocity profile, but the effects of diffusion are sensitive to length : diameter ratio.

\subsection{Response to temperature disturbances}

The proposed method for dealing with flow-determined transients, namely the longitudinal discretization of the conduit into a sequence of well-mixed nodes, leads naturally to a first-order energy balance on the fluid in each node:

$$
C_{a} \frac{d T_{i}}{d t}=\dot{m} c_{p}\left(T_{i-1}-T_{i}\right)-h_{c} A_{i}\left(T_{i}-T_{w, i}\right)
$$

The majority of conduits are constructed from a metal inner layer, with or without insulation and an outer protective layer. In terms of dynamic response to a disturbance from the inside, in general the Biot number of a metal conduit wall is low

$$
N_{B i}=\frac{h_{c} D}{k}
$$

indicating that quite small errors will be introduced by adopting a lumped thermal capacitance for the conduit wall. Here the characteristic dimension $D$ is taken as the thickness of the inner lining 


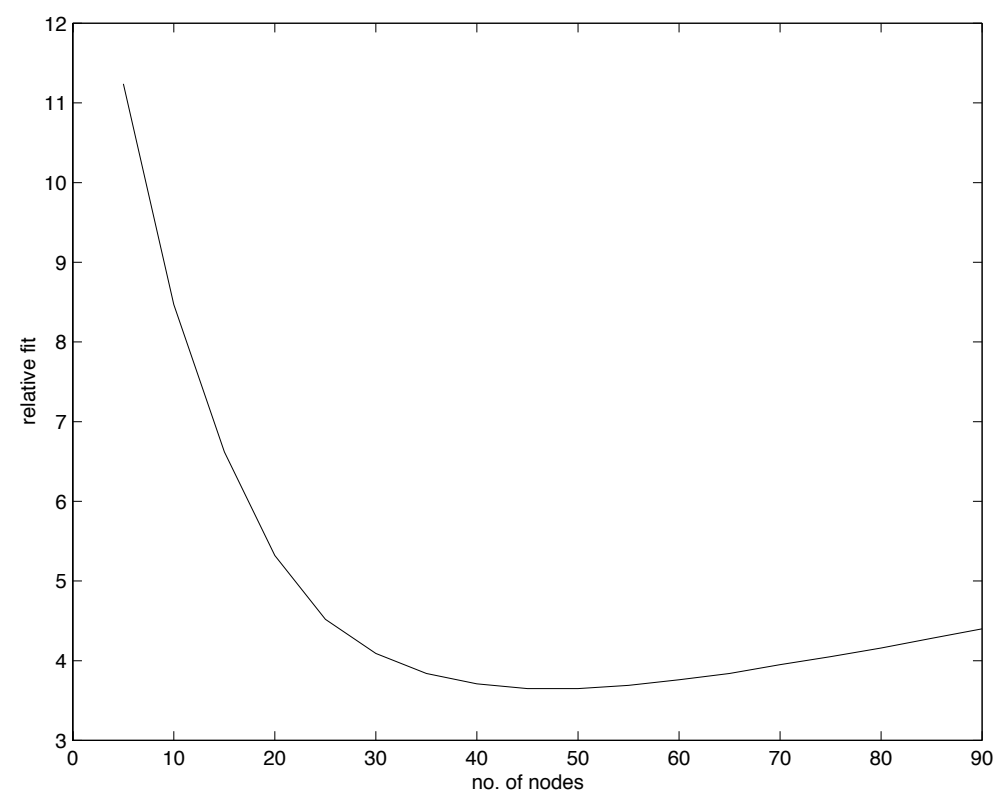

Figure 4: Optimal discretization of conduit

of the conduit.

A prototype duct was available for empirical validation purposes, with standard refrigeration construction consisting of inner and outer steel skins $1 \mathrm{~mm}$ thick, separated by $50 \mathrm{~mm}$ insulation. The prototype was a component of a climatic wind tunnel for which a dynamic model was being developed for testing improved control strategies (Roberts 2000). The climatic wind tunnel is used for vehicle testing and the duct formed part of the air handling plant serving a soak room in which vehicles were pre-conditioned. The relevant thermal properties are summarized in Table 1.

\begin{tabular}{|l|l|l|l|}
\hline material & $k\left(\mathrm{~W} \mathrm{~m}^{-1} \mathrm{~K}^{-1}\right)$ & $\rho\left(\mathrm{kg} \mathrm{m}^{-3}\right)$ & $c_{p}\left(J \mathrm{~kg}^{-1} \mathrm{~K}^{-1}\right)$ \\
\hline steel & 60.5 & 7854.0 & 434.0 \\
\hline insulation & 0.026 & 70.0 & 1045.0 \\
\hline
\end{tabular}

Table 1: Thermal properties of duct materials

The inside film heat transfer coefficient was calculated from a standard relationship for forced convection in ducts (Incropera and De Witt 1990)

$$
N_{N u}=0.023\left(N_{R e}\right)^{0.8}\left(N_{P r}\right)^{0.4}
$$

and the outside heat transfer coefficient from empirical data for convection and radiation heat transfer from pipes in still air (McAdams 1954).

A benchmark finite difference model of this construction was set up with capacitance nodes in each of the steel layers, together with three capacitance nodes in the insulation. This was compared with a simple ' $\mathrm{T}$ ' model which only considered the thermal capacitance of the inner steel layer. The imposed disturbance was a step rise in internal fluid temperature of $1 \mathrm{~K}$, and the calculated 


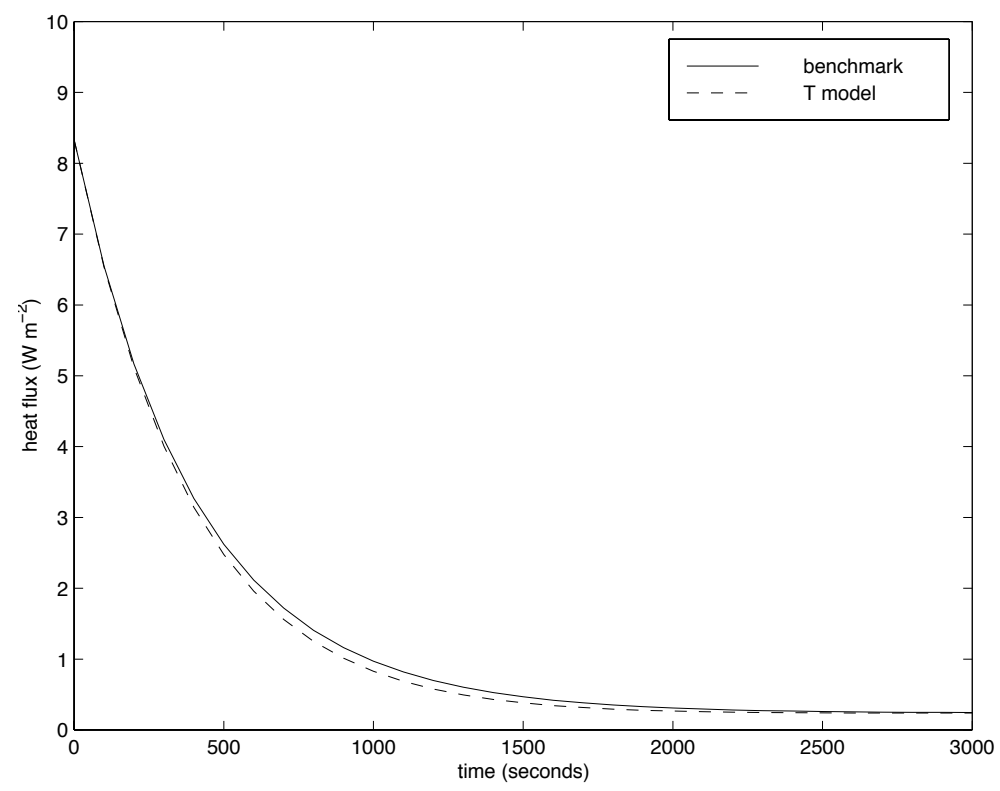

Figure 5: Thermal response of conduit walls

response was the heat flux into the inner surface.

A comparison of these two models is shown in Fig 5 which shows very small differences in the predicted response of the two models. It was concluded that the simple, ' $\mathrm{T}$ ' model gave an adequate representation of the thermal response of the conduit wall.

\subsection{Combined fluid and thermal model}

For each discretized conduit section, the combination of a well-mixed flow regime and a simple lumped parameter representation of the conduit walls produced the second-order model configuration shown in Fig 6.

Each node consisted of a lumped fluid thermal capacitance element $C_{a}$, a conductance between the fluid and the inner layer $h_{i}$ (the thermal resistance of the steel layer is neglected), the thermal capacitance of the inner layer $C_{w}$ and a conductance $h_{w}$ (insulation and external film coefficient) between the wall capacitance and the outside.

This model could be incorporated in a wide range of modular simulation environments. In this study, four simple components (fluid node, thermal capacitance, conductance and Kirchoff heat flux node) were written in Neutral Model Format (NMF) (Sahlin and Sowell 1989), combined to form discretized conduit sections, then solved using a commercial modular simulation environment (Bris Data 1998). 


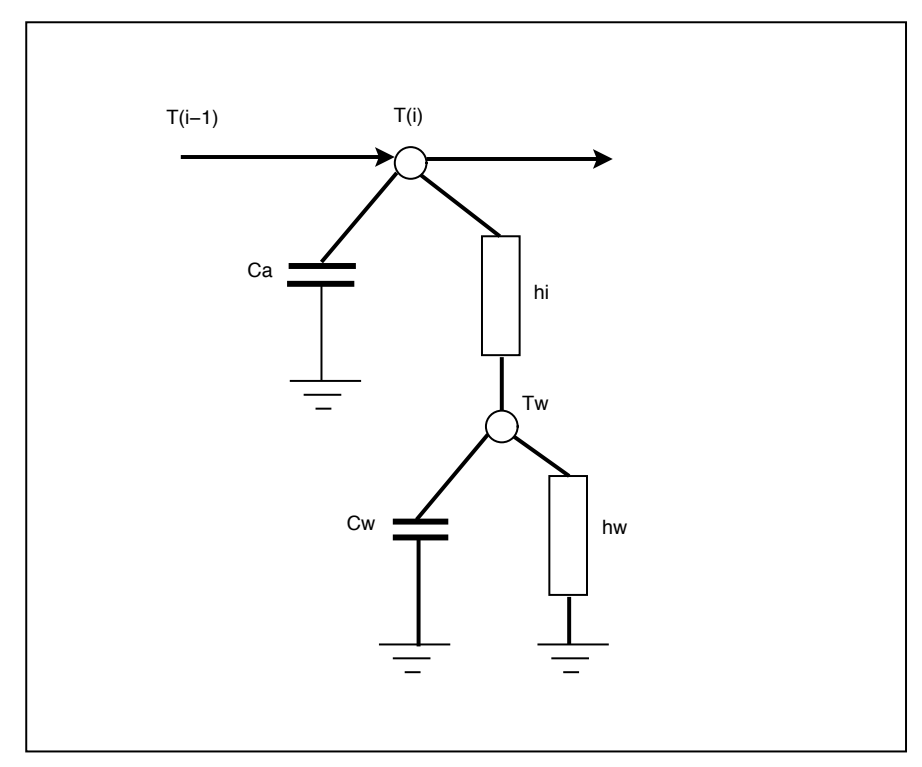

Figure 6: Schematic of discretized duct model

\section{Output and validation}

\subsection{Model response — flow dynamics}

The model was firstly used to calculate the response of the prototype duct to a step change in entering fluid temperature for varying numbers of well-mixed nodes. The duct was $21.2 \mathrm{~m}$ long and had a cross-sectional area of $0.64 \mathrm{~m}^{2}$. Under normal operating conditions it had a mean residence time of around 3 seconds and the walls had a thermal time constant of approximately 200 seconds: the response was therefore considered over these two time scales.

In the interests of generality, all results are presented in terms of outlet fluid reduced temperature

$$
\theta=\frac{T-T_{\text {init }}}{T_{s s}-T_{\text {init }}}
$$

as a function of dimensionless time

$$
\tau=\frac{\dot{\nu} t}{V}
$$

The response of the conduit model to a step change in inlet air temperature is shown in Fig 7 for varying levels of duct discretization.

The distinction between the short-term transients caused by the flow and the longer-term effects of thermal capacitance are evident. It can be seen that around 20 nodes gave a clear representation of the flow time delay and that the dynamics caused by the fluid flow have effectively disappeared after $\tau=3$ (i.e. three 'flush times'). 


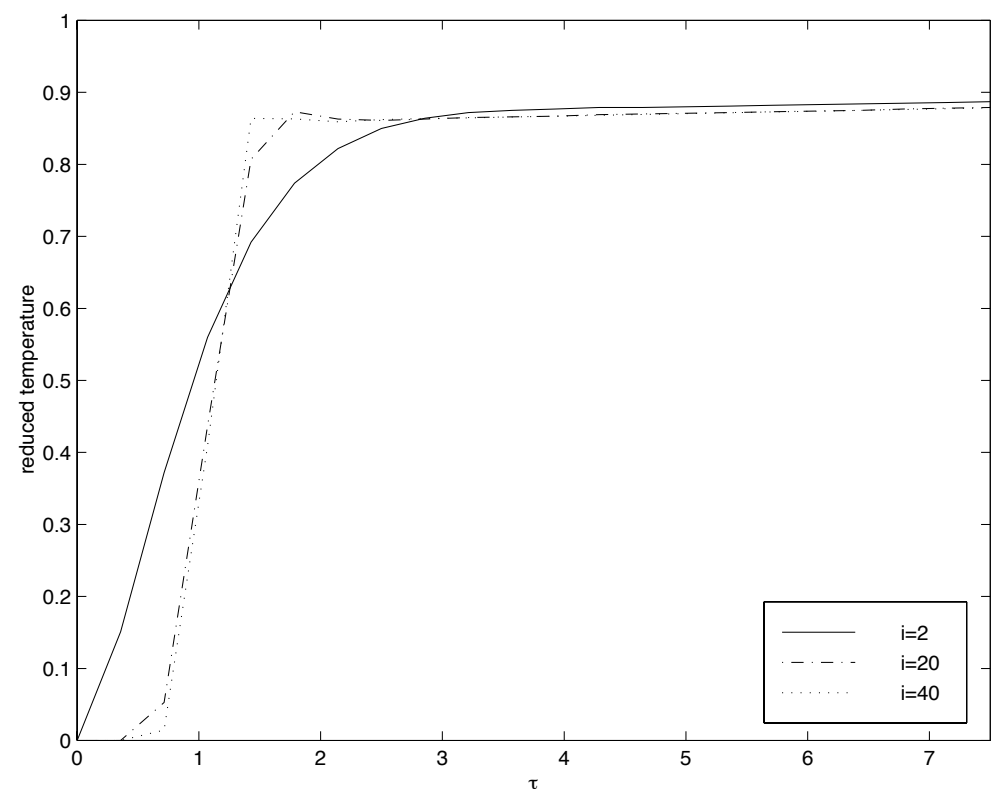

Figure 7: Temperature response of nodal conduit model

\subsection{Inter-model comparison}

The experimental determination of residence time distribution in a small volume such as a duct is difficult to carry out on an intensively used plant, hence the evaluation of the nodal model's short-term response was carried out by inter-model comparison. The comparators selected were the simplified time delay/first-order model of Clark, the transfer function model of Tobias and the TRNSYS pipe/duct model Type 31 (all described previously).

The models were configured with the same parameters and so had identical steady-state operation. The imposed disturbance was again a step rise in input air temperature and the results expressed in dimensionless terms: the results of this test are shown in Fig 8. The distinction between flow transients and thermal transients is immediately evident, with all the models showing a time delay of approximately $\tau=1$. However, the nodal model is the only one to show a response at less than one 'flush time': this is to be expected as it takes into account the radial velocity profile in the duct.

The transfer function model predicts a small step rise in temperature, with a rather faster rise than the nodal model in the thermally dominated regime. The simple time delay/first order model does not predict a sharp initial temperature rise, although this is somewhat compensated for by its smaller time constant. Configured for this prototype, the Type 31 model essentially reaches the steady-state condition immediately after the time delay, as this model accounts for the thermal capacitance of the fluid (in this case air) and not that of the duct wall.

Due to the modular nature of the nodal model, it was a fairly straightforward matter to insert a longitudinal conduction link into this model, to account for conduction along the inner lining of the duct. In none of the configurations tried did the longitudinal heat flux exceed $0.1 \%$ of the radial value, hence this was not incorporated subsequently in the nodal model. 


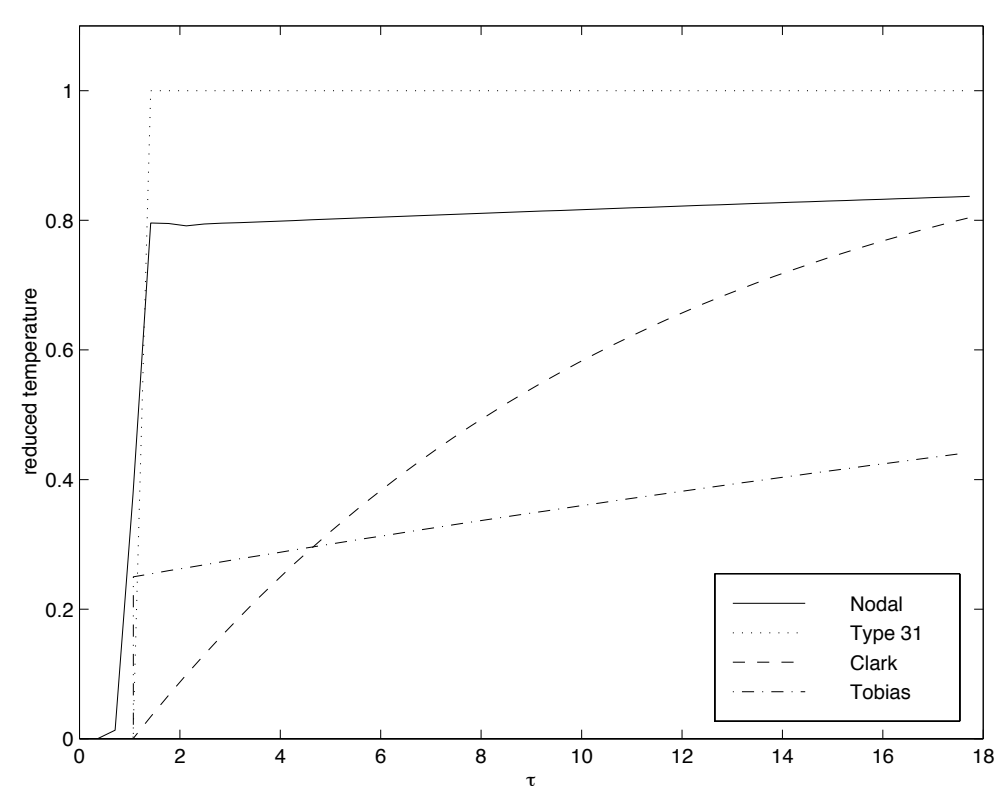

Figure 8: Inter-model comparison of step input

\subsection{Empirical test}

In the prototype plant it was possible to manipulate the control system to impose sudden small changes in the inlet temperature of the air: however, this could not be carried out in such a way as to provide a reliable indication of the short-term response caused by the flow dynamics. The disturbance was, however, sufficiently rapid to serve as a comparison of the thermal transients. The inlet and outlet air temperatures in the duct were measured by arrays of four T1/T2 thermocouples using an electronic reference junction. Fig 9 shows the results of imposing a sudden reduction in inlet air temperature followed by a rise approximately 60 seconds later: the inputs to the models were the measured inlet air temperature and volume flow rate, determined by a Pitot-tube traverse. The initial outlet steady-state temperature was $15.7{ }^{\circ} \mathrm{C}$.

The results are presented here as actual exit air temperature against elapsed time in seconds and a comparison with the TRNSYS Type 31 is not shown as this model, with air as the working fluid, has effectively no thermal transients.

The nodal model satisfactorily predicted the exit temperature of the air, including the 'plateau' at around 140 seconds caused by a delay in the control system: the maximum error observed in this time sequence was $0.3{ }^{\circ} \mathrm{C}$. The time delay/first order model of Clark had effectively too much thermal inertia at this time scale, whereas the transfer function model of Tobias over-predicted the swings in temperature.

\section{Conclusion}

A dynamic model for the thermal response of duct/pipe systems is described, based on discretization of the duct into well-mixed nodes. The model can be used to study the effects of variations 


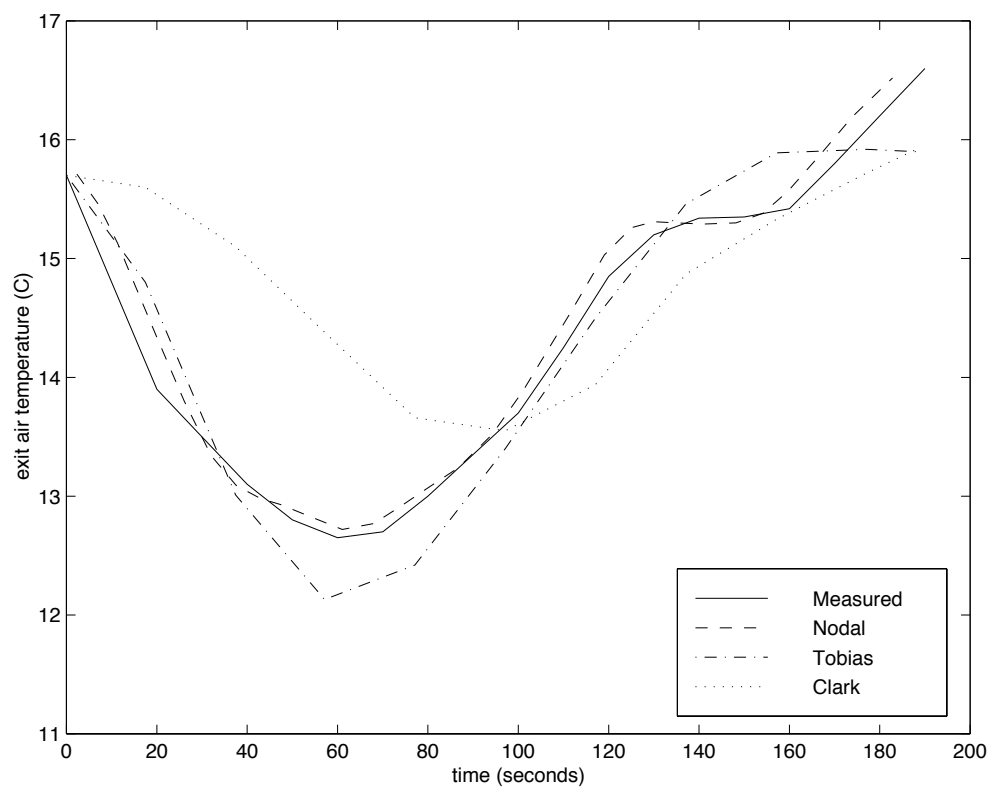

Figure 9: Measured and calculated temperature response

in flow rate, input temperature and pollutant concentration on the outlet conditions of the conduit. Consideration of the residence time distribution in a conduit with fully-developed turbulent flow, calculated from the radial velocity profile and eddy diffusivity, indicates an optimal level of discretization of 46 nodes, although satisfactory performance should be obtained with around 20 . This result is substantially independent of the length:diameter ratio of the conduit.

The method generates a response which includes a characteristic time delay but does not need explicit access to the system time in a dynamic simulation, making it applicable within a wide range of simulation environments. If the simulation program internally generates the time step, the resulting dynamics should appear automatically. If the time step is user-specified, then it must be set to a rather lower value than the mean residence time of the fluid in a conduit section.

A comparison of the short-term (flow dominated) response has been made with three published dynamic duct models: the nodal model shows the expected steep initial response (caused by the fluid flow characteristics), followed by a more gradual temperature rise due to the thermal dynamics of the conduit material.

The response of the model in the thermal regime was compared with experimental test results where rapid changes were made in to the inlet temperature of a steel-lined, insulated duct of standard refrigeration construction. A model consisting of 40 well-mixed nodes in series predicted the outlet air temperature of the duct to within a maximum error of $0.3^{\circ} \mathrm{C}$. Much larger errors were obtained with predictions from existing models. 


\section{Nomenclature}

\begin{tabular}{|c|c|c|}
\hline$a$ & polynomial coefficient & - \\
\hline$A$ & surface area & $m^{2}$ \\
\hline$c_{p}$ & specific heat (constant pressure) & $J k g^{-1} K^{-1}$ \\
\hline$C_{a}$ & fluid thermal capacitance & $J k g^{-1} K^{-1}$ \\
\hline$C_{w}$ & conduit wall thermal capacitance & $J k g^{-1} K^{-1}$ \\
\hline$D$ & characteristic length & $m$ \\
\hline$F$ & fraction of passive fluid thermal property & - \\
\hline$h_{c}$ & convective heat transfer coefficient & $W m^{-2} K^{-1}$ \\
\hline$h_{i}$ & inner surface heat transfer coefficient & $W m^{-2} K^{-1}$ \\
\hline$h_{o}$ & outer surface heat transfer coefficient & $W m^{-2} K^{-1}$ \\
\hline$h_{w}$ & wall/outer surface heat transfer coefficient & $W m^{-2} K^{-1}$ \\
\hline$i$ & integer & - \\
\hline$k$ & thermal conductivity & $W m^{-1} K^{-1}$ \\
\hline$L$ & length & $m$ \\
\hline$\dot{m}$ & fluid mass flow rate & $k g s^{-1}$ \\
\hline$n$ & integer & - \\
\hline$N_{B i}$ & Biot number & - \\
\hline$N_{P r}$ & Prandtl number & - \\
\hline$N_{R e}$ & Reynolds number & - \\
\hline$r$ & relative radial position & - \\
\hline$R$ & duct radius & $m$ \\
\hline$t$ & time & $s$ \\
\hline$t_{c}$ & time constant & $s$ \\
\hline$t_{d}$ & time delay & $s$ \\
\hline$T$ & temperature & $C$ \\
\hline$T_{i n}$ & fluid inlet temperature & $C$ \\
\hline$T_{\text {init }}$ & fluid initial temperature & $C$ \\
\hline$T_{\text {out }}$ & fluid outlet temperature & $C$ \\
\hline$T_{s s}$ & steady-state fluid temperature & $C$ \\
\hline$T_{w}$ & wall temperature & $C$ \\
\hline$u$ & longitudinal velocity & $m s^{-1}$ \\
\hline$U$ & overall heat transfer coefficient & $W m^{-2} K^{-1}$ \\
\hline$V$ & system volume & $m^{3}$ \\
\hline$x$ & longitudinal distance & $m$ \\
\hline$\beta$ & diffusivity correction & - \\
\hline$\rho$ & density & $k g \mathrm{~m}^{3}$ \\
\hline$\theta$ & reduced temperature & - \\
\hline$\tau$ & dimensionless time & - \\
\hline$\dot{\nu}$ & volume flow rate & $m^{3} s^{-1}$ \\
\hline
\end{tabular}

\section{$7 \quad$ References}

Bourdouxhe, J-P., M. Grodent, and J. Lebrun. 1998. Reference guide for dynamic models of HVAC equipment. Atlanta: American Society of Heating, Refrigerating and Air-Conditioning Engineers. 
Tobias, J. R. 1973. Simplified transfer function for temperature response of fluids flowing through coils, pipes or ducts. ASHRAE Transactions 79(1):19-22.

Park C., D. R. Clarke and G. E. Kelly, 1985. An overview of HVACSIM+, a dynamic building/HVAC/control simulation program. Proceedings of the First Annual Building Energy Simulation Conference, IBPSA, Seattle, WA.

Clark, D. R., C.R. Hill and C.W. Hurley, 1985. Dynamic models for HVAC system components. ASHRAE Transactions 91(1B):737 - 751 .

Klein, S. et al., 1996. Type 31: pipe or duct TRNSYS Reference Manual:4.5.4-1 - 4.5.4-3, Solar Energy Laboratory, University of Wisconsin-Madison, Madison, USA.

Escudie, J-C. and L. Laret, 1994. CSTBat, a simulation tool for building and heating system analysis within TRNSYS. Proceedings of System Simulation '94:403 - 424. University of Liège, Belgium.

Danckwerts, P. V., 1953. Continuous flow systems. Chemical Engineering Science, 2(1):1 - 13.

Bosworth, R. C. L., 1949. Phil. Mag. 39:847 - 850.

Sahlin, P. and E. F. Sowell, 1989. A neutral model format for building simulation models. Proceedings of Building Simulation '89, IBPSA, Vancouver.

Bris Data 1998. Technical literature on IDA Solver, Bris Data AB, Vasterlanggata 27, 11129 Stockholm, Sweden.

Incropera, F. P. and D. de Witt 1990. Introduction to heat transfer. New York:Wiley.

McAdams, W. H. 1954. Heat transmission. New York:McGraw-Hill.

Roberts, E C, 2000. Energy simulation of climatic wind tunnel plant. PhD thesis, Loughborough University, UK. 